\title{
A Comparison of Two Equivalent Real Formulations for Complex-Valued Linear Systems Part 1: Introduction and Method
}

\author{
Abnita Munankarmy and Michael A. Heroux \\ Department of Computer Science \\ College of Saint Benedict \\ 37 South College Avenue \\ St. Joseph, Minnesota 56374 USA
}

Received May 14, 2002

Accepted October 22, 2002

\begin{abstract}
Many iterative linear solver packages focus on real-valued systems and do not deal well with complex-valued systems, even though preconditioned iterative methods typically apply to both real and complex-valued linear systems. Instead, commonly available packages such as PETSC [1] and Aztec [2] tend to focus on the real-valued systems, while complex-valued systems are seen as a late addition. At the same time, by changing the complex problem into an equivalent real formulation (ERF), a real valued solver can be used. In this paper we consider two ERF's that can be used to solve complex-valued linear systems. We investigate the spectral properties of each and show how each can be preconditioned to move eigenvalues in a cloud around the point $(1,0)$ in the complex plane. Finally, we consider an interleaved formulation, combining each of the previously mentioned approaches, and show that the interleaved form achieves a better outcome than either separate ERF.
\end{abstract}

\section{INTRODUCTION}

This paper describes an effective extension of a real-valued preconditioned iterative solver package for complex-valued linear systems

$$
C w=d,
$$

where $C$ is an m-by-n complex matrix, $d$ is a known n-by-1vector and $w$ is an unknown nby- 1 vector. There are few complex-valued solver packages available to solve this system. Here we solve this system using an equivalent real formulation (ERF). This paper explores several topics left for further research in the paper by Day and Heroux [3], who first introduced the topic.

\section{PROPECTIVE ERFs}

In order to derive ERFs for equation (1), we begin by writing (1) in terms of its real and imaginary parts. Specifically,

$$
(A+i B)(x+i y)=b+i c
$$

where $C=A+i B, w=x+i y$ and $d=b+i c$. Doing this, we can consider four possible 2by-2 block formulations as described by Day and Heroux [3]. These are shown below in equations (2a) - (2d), and their solutions are equivalent to those for equation (1). We shall denote these formulations by $\mathrm{K} 1, \mathrm{~K} 2$, $\mathrm{K} 3$, and $\mathrm{K} 4$.

$\mathrm{K} 1$ formulation,

$$
\left(\begin{array}{cc}
A & -B \\
B & A
\end{array}\right)\left(\begin{array}{l}
x \\
y
\end{array}\right)=\left(\begin{array}{l}
b \\
c
\end{array}\right)
$$

K2 formulation,

$$
\left(\begin{array}{cc}
A & B \\
B & -A
\end{array}\right)\left(\begin{array}{c}
x \\
-y
\end{array}\right)=\left(\begin{array}{l}
b \\
c
\end{array}\right)
$$

K3 formulation,

$$
\left(\begin{array}{cc}
B & A \\
A & -B
\end{array}\right)\left(\begin{array}{l}
x \\
y
\end{array}\right)=\left(\begin{array}{l}
c \\
b
\end{array}\right)
$$


and the K4 formulation,

$$
\left(\begin{array}{cc}
B & -A \\
A & B
\end{array}\right)\left(\begin{array}{c}
x \\
-y
\end{array}\right)=\left(\begin{array}{l}
c \\
b
\end{array}\right)
$$

For future reference, we shall denote the matrix associated with the $\mathrm{K} 1$ to $\mathrm{K} 4$ formulations by $K_{1}$ to $K_{4}$, respectively. Along with these four real formulations, there is also a combined formulation $\mathrm{K} 14$, which is expressed as an interleaved ERF. In the future, the matrix associated with $\mathrm{K} 14$ will be denoted by $K_{14}$. We will discuss $K_{14}$ in Section IV.

The eigenvalues of the matrices in the $\mathrm{K} 2$ and $\mathrm{K} 3$ formulations are in problematic configuration because any hull containing the spectra necessarily contains the origin, which degrades the convergence rate of an iterative method such as GMRES [5]. The other reasons for this are beyond the scope of this paper. Matrices in the K1 and K4 formulations are more promising because they have more favorable spectral properties, and can be preconditioned to move eigenvalues in a cloud around the point $(1,0)$ in the complex plane. This makes the convergence rate of an iterative method, such as GMRES [5], more robust. It also impacts on the reflection of the eigenvalues of the complex matrix and the ERFs $\mathrm{K} 1, \mathrm{~K} 4$ and $\mathrm{K} 14$ respectively. Table 1 summarizes the spectral properties of $K_{1}$ to $\mathrm{K}_{14}$. Since $\mathrm{K} 1$ and $\mathrm{K} 4$ formulations are more promising than $\mathrm{K} 2$ and $\mathrm{K} 3$, we want to determine if either $\mathrm{K} 1$ or $\mathrm{K} 4$ would be the best one to find a solution. Success with the $\mathrm{K} 1$ and $\mathrm{K} 4$ formulations depends on the quality of the preconditioner but the slower rate is due to the spectral properties of the ERF.

For the $\mathrm{K} 1$ formulation, the spectrum of $K_{1}$ should not present a major dilemma to an iterative method such as GMRES, given all the eigenvalues of $C$ are on one side of the imaginary axis. However, $\mathrm{K}_{1}$ will have twice as many problematic eigenvalues if $\mathrm{C}$ has eigenvalues on both sides of the imaginary axis and this is a property that degrades the GMRES convergence rate. Most importantly, the convex hull containing the eigenvalues of $\mathrm{K}_{1}$ will also contain the origin. For the $\mathrm{K} 4$ formulation, the eigenvalues of $\mathrm{K}_{4}$ will be in the right half plane as long as all the eigenvalues of $\mathrm{C}$ are in the upper half plane. The K4 formulation is actually a constant variation of the $\mathrm{K} 1$ formulation.

It has been shown [3] that if $C$ is Hermitian then $K_{1}$ is symmetric and the convergence rate of an ERF is identical to the convergence rate with the original complex formulation. However, if this is not the case there are different results based on the eigenvalues of the complex matrix. This will be demonstrated below.

\section{BASIC PROPERTIES OF ERFS}

In this section we present a simple example to illustrate the important properties of each ERF. This example illustrates the distribution of the eigenvalues of a complex matrix $C$ that has eigenvalues on one side of the imaginary axis, and then shows how that impacts the eigenvalues distribution of $\mathrm{K}_{1}$, $\mathrm{K}_{4}$, and $\mathrm{K}_{14}$, respectively.

The original matrix $\mathrm{C}$ is a tridiagonal matrix of the form

$$
C=\left[\begin{array}{cccccccccc}
2+i & 2+i & 0 & 0 & 0 & 0 & 0 & 0 & 0 & 0 \\
1+\frac{1}{2} i & 4+2 i & 3+\frac{3}{2} i & 0 & 0 & 0 & 0 & 0 & 0 & 0 \\
0 & 2+i & 6+3 i & 4+2 i & 0 & 0 & 0 & 0 & 0 & 0 \\
0 & 0 & 3+\frac{3}{2} i & 8+4 i & 5+\frac{5}{2} i & 0 & 0 & 0 & 0 & 0 \\
0 & 0 & 0 & 4+2 i & 10+5 i & 6+3 i & 0 & 0 & 0 & 0 \\
0 & 0 & 0 & 0 & 5+\frac{5}{2} i & 12+6 i & 7+\frac{7}{2} i & 0 & 0 & 0 \\
0 & 0 & 0 & 0 & 0 & 6+3 i & 14+7 i & 8+4 i & 0 & 0 \\
0 & 0 & 0 & 0 & 0 & 0 & 7+\frac{7}{2} i & 16+8 i & 9+\frac{9}{2} i & 0 \\
0 & 0 & 0 & 0 & 0 & 0 & 0 & 8+4 i & 18+9 i & 10+5 i \\
0 & 0 & 0 & 0 & 0 & 0 & 0 & 0 & 9+\frac{9}{2} i & 20+10 i
\end{array}\right]
$$


Figures 1, 2 and 3 show scatter plots of the eigenvalues for the matrix $C$ and its $K 1$ and K4 formulations. Denoting the spectrum of a matrix $A$ by $\sigma(A)$, we see from these figures that $\sigma\left(\mathrm{K}_{1}\right)$ contains the $\sigma(\mathrm{C})$ and the reflection of $\sigma(\mathrm{C})$ across the real axis. $\sigma\left(\mathrm{K}_{4}\right)$ is obtained from the $\sigma(\mathrm{C})$ by rotating $\sigma(\mathrm{C})$ by 90 degrees clockwise and then reflecting across the real axis. For the K14 formulation, the eigenvalues of the matrix $\mathrm{K}_{14}$ act the same way as those of matrix $\mathrm{K}_{1}$.

In general, we can summarize the spectral properties for the $\mathrm{K} 1$ through $\mathrm{K} 4$ formulations using Table 1, reproduced from [3].

\begin{tabular}{|c|c|c|}
\hline Matrix & & Spectral Properties \\
\hline $\mathrm{K}_{1}$ & $\begin{array}{l}\text { (i) } \\
\text { (ii) }\end{array}$ & $\begin{array}{l}\text { If } \lambda \in \sigma(C) \text { then } \lambda, \lambda^{-1} \in \sigma\left(K_{1}\right) . \\
\text { If } C \text { is Hermitian (positive definite) then } K_{1} \text { is symmetric (positive definite). }\end{array}$ \\
\hline $\mathrm{K}_{2}$ & $\begin{array}{l}\text { (i) } \\
\text { (ii) }\end{array}$ & $\begin{array}{l}\text { If } \lambda \in \sigma\left(\mathrm{K}_{2}\right) \text { then }-\lambda, \lambda^{-1},-\lambda^{-1} \in \sigma\left(\mathrm{K}_{2}\right) \\
\text { If } \mathrm{C} \text { is symmetric then } \mathrm{K}_{2} \text { is symmetric. }\end{array}$ \\
\hline $\mathrm{K}_{3}$ & $\begin{array}{l}\text { (i) } \\
\text { (ii) } \\
\text { (iii) }\end{array}$ & $\begin{array}{l}\text { If } \lambda \in \sigma\left(\mathrm{K}_{3}\right) \text { then }-\lambda, \lambda^{-1},-\lambda^{-1} \in \sigma\left(\mathrm{K}_{3}\right) \\
\text { If } C \text { is symmetric then } \mathrm{K}_{3} \text { is symmetric. } \\
\sigma\left(\mathrm{K}_{3}\right)=\sigma\left(\mathrm{K}_{2}\right)\end{array}$ \\
\hline $\mathrm{K}_{4}$ & $\begin{array}{l}\text { (i) } \\
\text { (ii) }\end{array}$ & $\begin{array}{l}\text { If } \lambda \in \sigma(C) \text { then }-i \lambda, i \lambda^{-1} \in \sigma\left(K_{4}\right) \text {. } \\
\text { If } C \text { is Hermitian (positive definite) then } K_{4} \text { is skew symmetric (with } \\
\text { eigenvalues that have positive imaginary parts). }\end{array}$ \\
\hline $\mathrm{K}_{14}$ & $\begin{array}{l}\text { (i) } \\
\text { (ii) } \\
\text { (iii) }\end{array}$ & $\begin{array}{l}\text { If } \lambda \in \sigma(C) \text { then } \lambda, \lambda^{-1} \in \sigma\left(K_{14}\right) \text {. } \\
\text { If } C \text { is Hermitian (positive definite) and the diagonal values have larger } \\
\text { real part, then } K_{14} \text { is symmetric (positive definite). } \\
\text { If } C \text { is Hermitian (positive definite) and the diagonal values have larger } \\
\text { imaginary part, then } K_{4} \text { is skew symmetric (with eigenvalues that have } \\
\text { positive imaginary parts). }\end{array}$ \\
\hline
\end{tabular}

Table 1. Spectral Properties of the $K$ formulations. $\sigma(K)$ denotes the spectrum of $K$ and $i=\sqrt{-1}$

\section{INTERLEAVED ERF}

From the example in section III, we can see intuitively that the $\mathrm{K} 1$ and $\mathrm{K} 4$ formulations will be desirable ERF's in a different setting. When $\sigma(C)$ tends to have eigenvalues with large real parts, K1 would tend to cluster the eigenvalues around $(1,0)$ in the complex plane. When these eigenvalues have large imaginary parts, K4 would be a better formulation. In this section we introduce the K14 formulation that attempts to provide a combined approach using a simple heuristic. In the K14 formulation, we interleave the individual $\mathrm{K} 1$ and $\mathrm{K} 4$ formulations for each equation of the complex matrix. Interleaving is done by permuting and scaling the real matrix. Permutation of the matrix is necessary for both theoretical and computational reasons. The process of interleaving is demonstrated below. Success with the K14 formulation depends on the real and imaginary parts of the eigenvalues. The major part of the solution depends on eigenvalues. Eigenvalues help to be familiar with the speed of the iterative method and the better the iterative method is, the better the solution. Success also depends on the quality of the preconditioner. In fact, our experience shows that for the classes of problems we are solving, in particular 
eigenvalues problems for computational fluid dynamics, interleaving leads to better diagonal precondtioners. The following example demonstrates an interleave process for a simple 2-by-2 complex matrix for form a K14 formulation.

Let $\mathrm{C} w=\mathrm{d}$ be a 2-by-2 complex system with $\mathrm{C}$ explicitly defined as follows:

$$
\left[\begin{array}{ll}
1+2 i & 3+i \\
4+2 i & 5+i
\end{array}\right]\left[\begin{array}{l}
w_{1} \\
w_{2}
\end{array}\right]=\left[\begin{array}{l}
d_{1} \\
d_{2}
\end{array}\right]
$$

Then the $\mathrm{K} 1$ and $\mathrm{K} 4$ formulations of the linear system are as follows:

For K1,

$$
\left[\begin{array}{cccc}
1 & -2 & 3 & -1 \\
2 & 1 & 1 & 3 \\
4 & -2 & 5 & -1 \\
2 & 4 & 1 & 5
\end{array}\right]\left[\begin{array}{l}
x_{1} \\
y_{1} \\
x_{2} \\
y_{2}
\end{array}\right]=\left[\begin{array}{l}
b_{1} \\
c_{1} \\
b_{2} \\
c_{2}
\end{array}\right] .
$$

Writing out this matrix equation as a linear system of equations gives,

$$
\begin{aligned}
& x_{1}-2 y_{1}+3 x_{2}-y_{2}=b_{1} \\
& 2 x_{1}+y_{1}+x_{2}+3 y_{2}=c_{1} \\
& 4 x_{1}-2 y_{1}+5 x_{2}-y_{2}=b_{2} \\
& 2 x_{1}+4 y_{1}+x_{2}+5 y_{2}=c_{2}
\end{aligned}
$$

For K4,

$$
\left[\begin{array}{cccc}
2 & -1 & 1 & -3 \\
1 & 2 & 3 & 1 \\
2 & -4 & 1 & -5 \\
4 & 2 & 5 & 1
\end{array}\right]\left[\begin{array}{c}
x_{1} \\
-y_{1} \\
x_{2} \\
-y_{2}
\end{array}\right]=\left[\begin{array}{l}
b_{1} \\
c_{1} \\
b_{2} \\
c_{2}
\end{array}\right]
$$

The system of linear equations for the K4 matrix formulation is

$$
\begin{aligned}
& 2 x_{1}+y_{1}+x_{2}+3 y_{2}=b_{1} \\
& x_{1}-2 y_{1}+3 x_{2}-y_{2}=c_{1} \\
& 2 x_{1}+4 y_{1}+x_{2}+5 y_{2}=b_{2} \\
& 4 x_{1}-2 y_{1}+5 x_{2}-y_{2}=c_{2}
\end{aligned}
$$

We now observe that both the formulations have the same linear solutions. It is always true that $\mathrm{K} 1$ and $\mathrm{K} 4$ formulations should have the same solutions, and it is also true that the K14 formulation should have exactly the same solutions as the $\mathrm{K} 1$ and the $\mathrm{K} 4$ formulations.

The K14 formulation interleaves the $\mathrm{K} 1$ and $\mathrm{K} 4$ formulations on an equation-byequation basis by looking at the diagonal entries of the complex matrix $C$. In particular, for each diagonal entry, if the imaginary part of the entry is greater then the real part, then we use K4 for that equation. Otherwise, we use the $\mathrm{K} 1$ formulation. For the example above, the $\mathrm{K} 14$ formulation is,

$$
\left[\begin{array}{cccc}
2 & -1 & 1 & 3 \\
1 & 2 & 3 & -1 \\
4 & 2 & 5 & -1 \\
2 & -4 & 1 & 5
\end{array}\right]\left[\begin{array}{c}
x_{1} \\
-y_{1} \\
x_{2} \\
y_{2}
\end{array}\right]=\left[\begin{array}{l}
c_{1} \\
b_{1} \\
b_{2} \\
c_{2}
\end{array}\right] .
$$

Writing out the system of equations for this matrix equation gives,

$$
\begin{aligned}
& 2 x_{1}+y_{1}+x_{2}+3 y_{2}=c_{1} \\
& x_{1}-2 y_{1}+3 x_{2}-y_{2}=b_{1} \\
& 4 x_{1}-2 y_{1}+5 x_{2}-y_{2}=b_{2} \\
& 2 x_{1}+4 y_{1}+x_{2}+5 y_{2}=c_{2}
\end{aligned}
$$

In examining the $\mathrm{K} 14$ formulation, we observe that the diagonal values are larger in size than the diagonal values in the individual formulations. When the diagonal values are larger, many preconditoners tend to be more effective. In fact, one could introduce a thesis for matrices with larger diagonal values and the effect of using the preconditioners such as Jacobi and Gaussian Elimination methods.

\section{PRECONDITIONING ERFS}

Preconditioning can be done in many different ways, and hence in trying to solve the original complex system in equation (1) via the $\mathrm{K} 1$ formulation in equation (2a), the most fascinating question is how to precondition $\mathrm{K}_{1}$. Standard realvalued preconditioners such as Jacobi, Gauss-Seidel or ILU applied directly to $\mathrm{K}_{1}$ are not robust enough for our needs. Furthermore, the ordering of the unknowns is unsuitable for sparse matrix operations related to factorization, particularly ILU preconditioning [3]. A good preconditioner makes the iterations of $\mathrm{K}$ formulations comparable to that of solving the original 


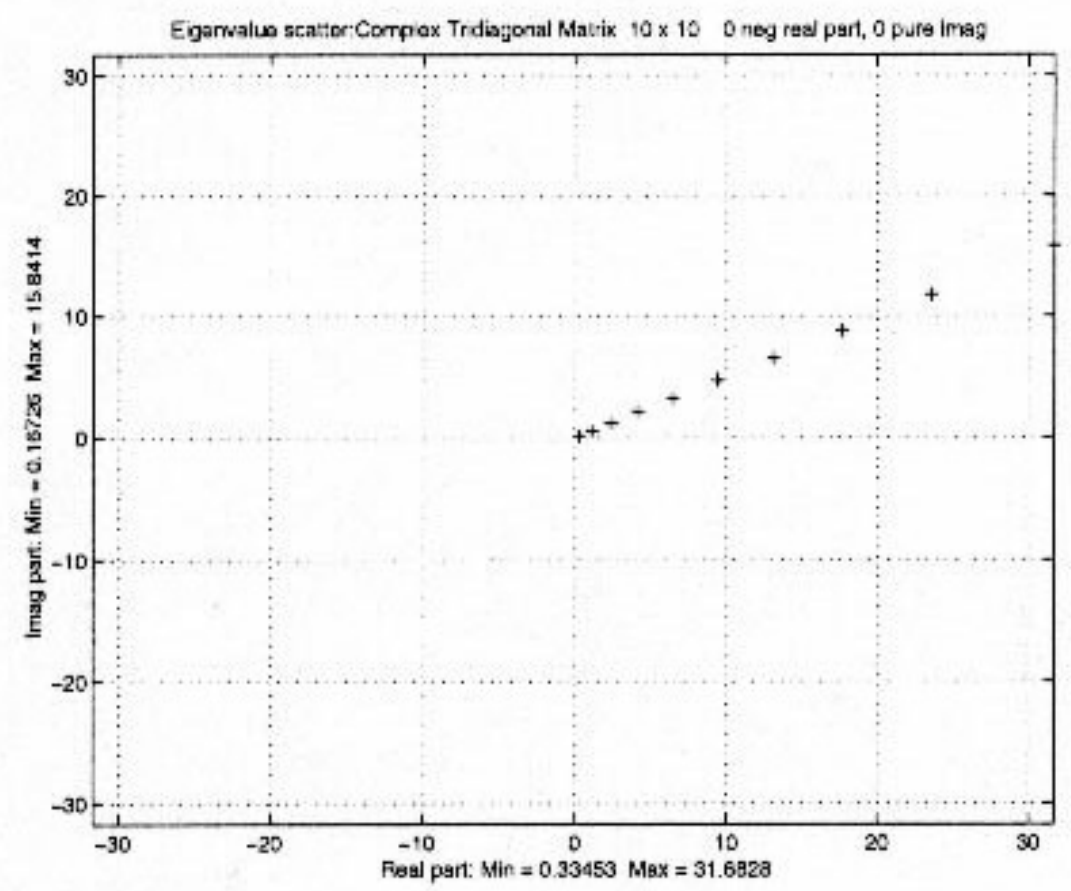

Figure 1. Eigenvalues of the complex tridiagonal matrix.

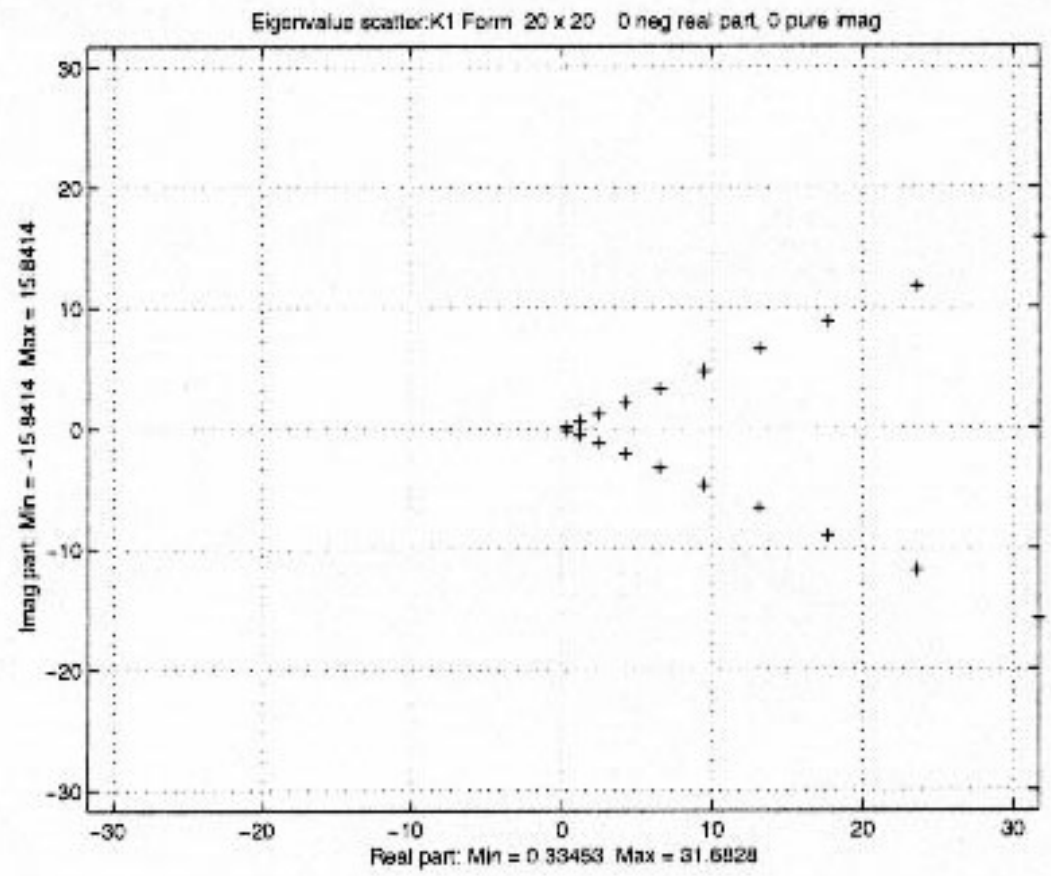

Figure 2. Eigenvalues of the $\mathrm{K} 1$ formulation matrix. 


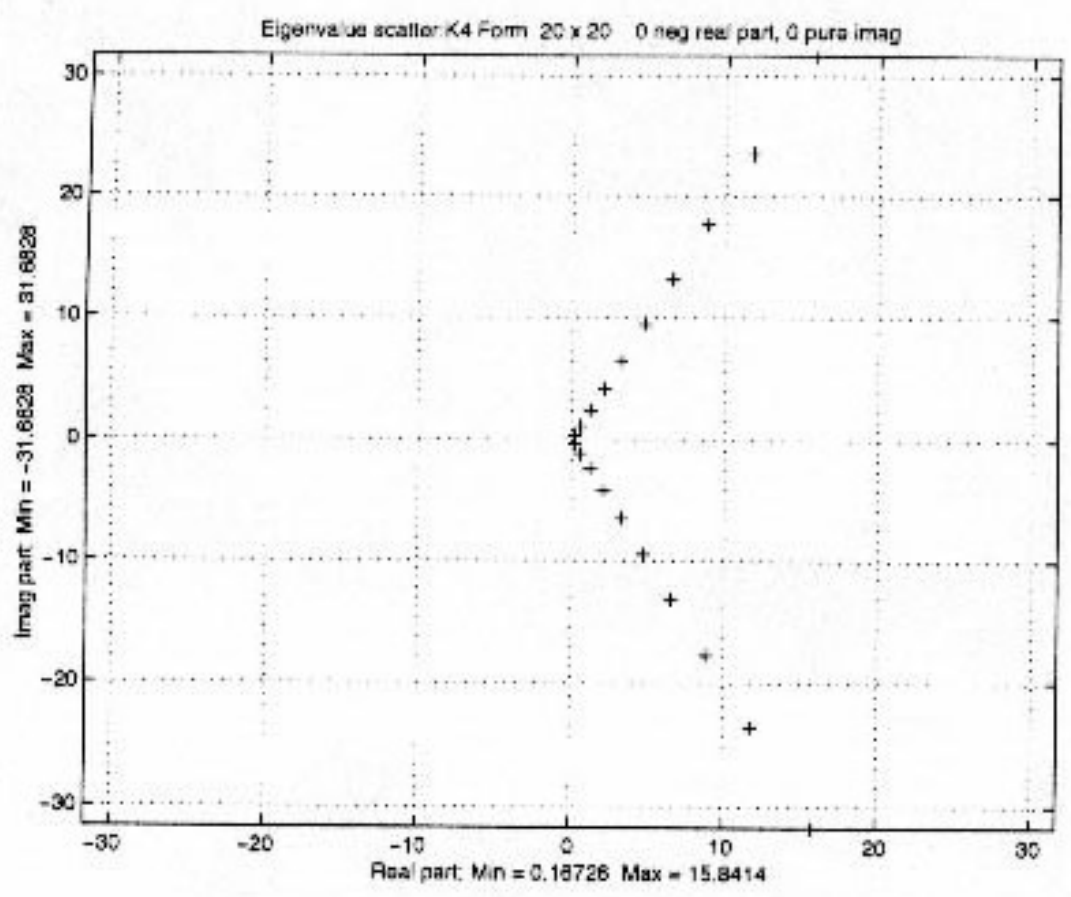

Figure 3. Eigenvalues of the K4 formulation matrix.

complex problem, with a true complex preconditioned iterative solver. Convergence often can be accelerated substantially by preconditioning, which can be thought of as implicitly multiplying matrix $A$ by matrix $M^{-1}$, where matrix $M$ is a preconditioner that we define. We start with a matrix equation

$$
A x=b,
$$

and assume that there exist a lower triangular matrix, $\mathrm{L}$, and an upper triangular matrix, $U$, such that $A=L U$. Further assuming that both $L$ and $U$ have inverses, equation (1) can be rewritten as follows,

$$
\begin{aligned}
& (L U) x=b \\
& (L U) I x=b \\
& (L U)\left(U^{-1} U\right) x=b
\end{aligned}
$$

where $\mathrm{I}$ is the identity matrix. Multiplying through by $\mathrm{L}^{-1}$ gives,

$$
L^{-1}(L U)\left(U^{-1} U\right) x=L^{-1} b
$$

or

$$
\left(L^{-1} L\right)\left(U U^{-1}\right) U x=L^{-1} b .
$$

Since $L^{-1} L=I$ and $U^{-1} U=I$, then

$$
U x=L^{-1} b,
$$

or, multiplying through with $\mathrm{U}^{-1}$,

$$
x=U^{-1} L^{-1} b .
$$

The goal of preconditioning is to find $L$ and $U$ such that $A \approx L U$. The better this approximation, the better the solution, equation (2), to equation (1).

For this research, we use the Incomplete LU (ILU) factorization preconditioner discussed by Saad [4]. This produces a lower triangular matrix, an upper triangular matrix, and a permutation matrix.

VI. FORMULATION OF $\mathrm{K}_{1}, \mathrm{~K}_{4}$, AND $\mathrm{K}_{14}$

The ILU factorization is often used for sparse matrices, and the approach that gives a better result is one that preserves the sparsity pattern of the complete matrix C. The entries of a block entry sparse matrix are all (small) dense (sub-) matrices. The $\mathrm{K}$ formulation preserves the non-zero 
pattern of the block entries with the effect of doubling the size of each dense sub-matrix.

In the $\mathrm{K}$ formulation, $\mathrm{c}_{\mathrm{pq}}=\mathrm{a}_{\mathrm{pq}}+i \mathrm{~b}_{\mathrm{pq}}$ corresponds, via the scalar $\mathrm{K} 1$ formulation, to the 2-by-2 block entry of the $2 m$-by- $2 n$ real matrix $\mathrm{K}$ given by

$$
\left(\begin{array}{cc}
a_{p q} & -b_{p q} \\
b_{p q} & a_{p q}
\end{array}\right)
$$

$$
C=\left(\begin{array}{ccccc}
c_{11} & 0 & c_{13} & 0 & c_{15} \\
0 & c_{22} & c_{23} & 0 & 0 \\
c_{31} & 0 & c_{33} & c_{34} & 0 \\
0 & 0 & c_{43} & c_{44} & 0 \\
c_{51} & 0 & 0 & 0 & c_{55}
\end{array}\right)
$$

then,

For example, if

$$
K=\left(\begin{array}{cccccccccc}
a_{11} & -b_{11} & 0 & 0 & a_{13} & -b_{13} & 0 & 0 & a_{15} & -b_{15} \\
b_{11} & a_{11} & 0 & 0 & b_{13} & a_{13} & 0 & 0 & b_{15} & a_{15} \\
0 & 0 & a_{22} & -b_{22} & a_{23} & -b_{23} & 0 & 0 & 0 & 0 \\
0 & 0 & b_{22} & a_{22} & b_{23} & a_{23} & 0 & 0 & 0 & 0 \\
a_{31} & -b_{31} & 0 & 0 & a_{33} & -b_{33} & a_{34} & -b_{44} & 0 & 0 \\
b_{31} & a_{31} & 0 & 0 & b_{33} & a_{33} & a_{34} & -b_{34} & 0 & 0 \\
0 & 0 & 0 & 0 & a_{43} & -b_{43} & a_{44} & -b_{44} & 0 & 0 \\
0 & 0 & 0 & 0 & b_{43} & a_{43} & a_{44} & -b_{44} & 0 & 0 \\
a_{51} & -b_{51} & 0 & 0 & 0 & 0 & 0 & 0 & a_{55} & -b_{55} \\
b_{51} & a_{51} & 0 & 0 & 0 & 0 & 0 & 0 & b_{55} & a_{55}
\end{array}\right)
$$

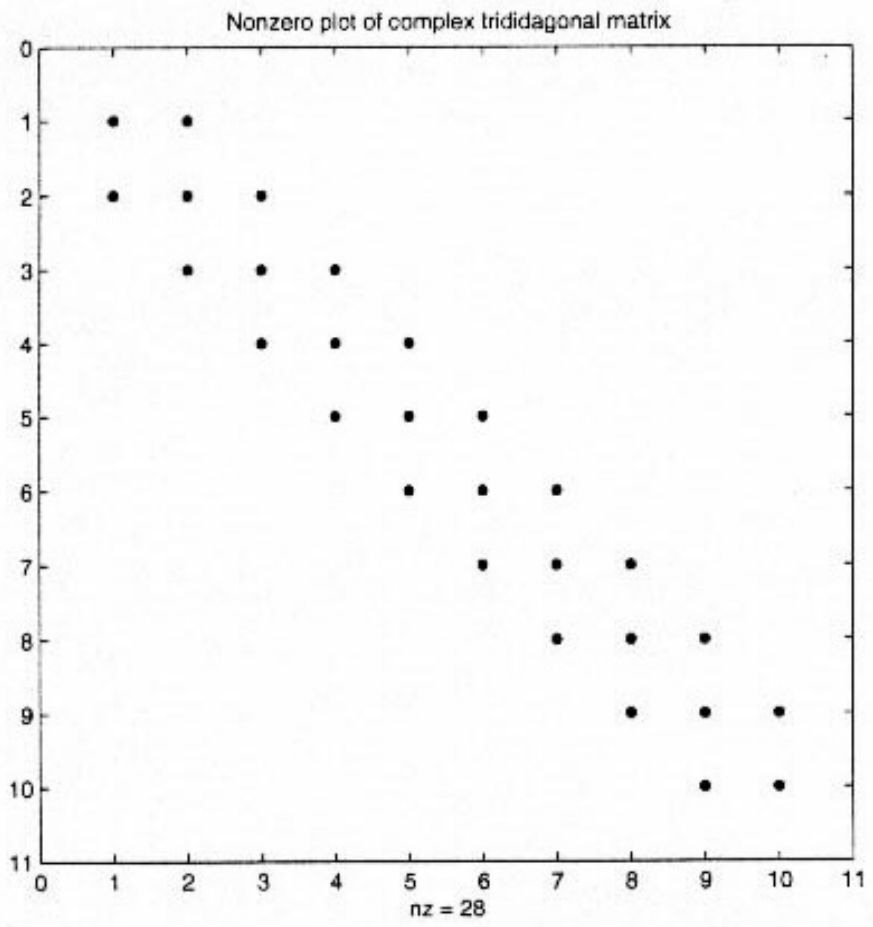

Figure 4. Non-zero pattern of a complex tridiagonal matrix. 
In the related real formulations, this is a way to preserve the non-zero structure in the block entries. We can see the tridiagonal pattern in Figure 4. We can generate the matrices $\mathrm{K}_{4}$ and $\mathrm{K}_{14}$ in a similar manner via the scalar K4 and K14 formulations, respectively. Figure 4 shows the non-zero pattern associated with the matrix $\mathrm{C}$ in Equation (3). Figure 5 shows the non-zero pattern that would be generated by any of the $\mathrm{K}$ formulations. Note how it preserves the sparsity pattern, but in block form.

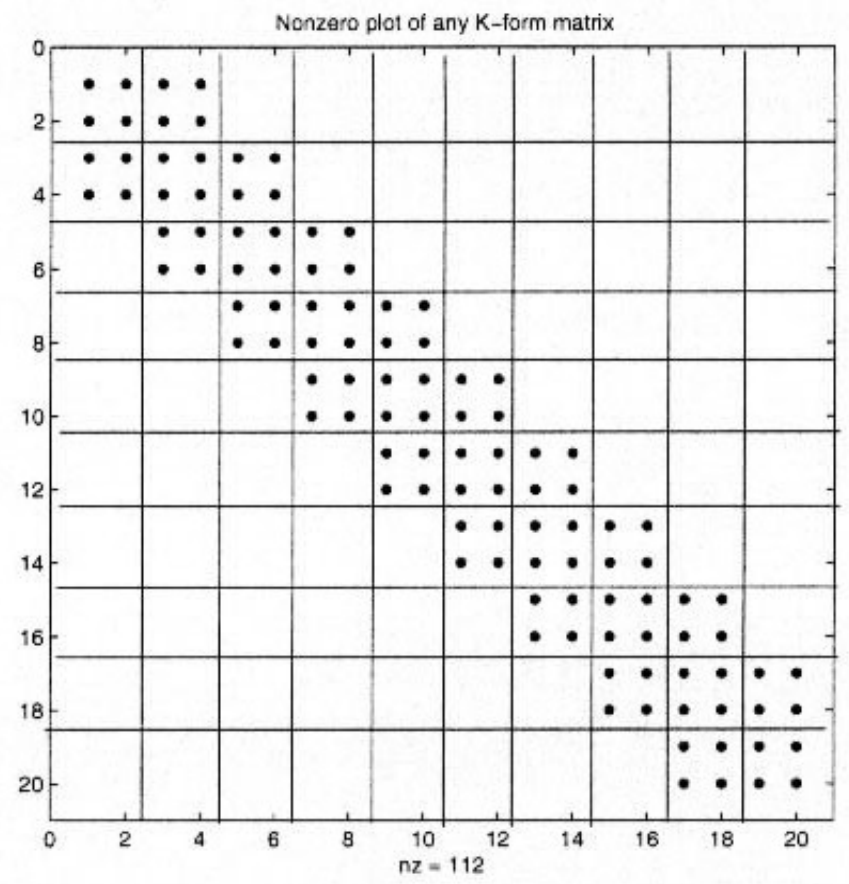

Figure 5. Non-zero pattern of any of the $\mathrm{K}$ formulations of the complex tridiagonal matrix.

\section{PRECONDITIONING IMPLEMENTATION}

The properties of the $\mathrm{K}$ formulation defined in section VI enable the preconditioned iterative solvers for complex linear systems to provide a better result. We can efficiently compute and apply the exact equivalent of a complex-valued preconditioner. $\mathrm{K} 1$ and $\mathrm{K} 4$ formulations have nice spectral properties; hence these formulations lead to convergence that is competitive with the true complex solver. Similarly, the properties of the interleaved formulation defined in section IV enable us to re-boost the solution. The K14 formulation can be used to obtain better ILU factors.

The command luinc, from the software MATLAB, was used to produce a unit lower triangular matrix, an upper triangular matrix, and a permutation matrix. We implemented the precondition in two ways. One way was by producing $L$ and $U$ from the original complex matrix, and using the same preconditioning to find the solutions for the $\mathrm{K} 1$ and $\mathrm{K} 4$ formulations. For example, let $C$ be the complex matrix. We get $L$ and $U$ from $[L, U]=\operatorname{luinc}\left(C, O^{\prime}\right)$. Transforming this into the $\mathrm{K} 1$ transformation of $L$ we get $L 1=K 1(L)$. What happens here is that the new $L 1$ is no longer a triangular matrix. We can see this in the following example:

Let $L$ be a 2-by-2 lower triangular matrix,

$$
L=\left(\begin{array}{ll}
I_{11} & 0 \\
I_{21} & I_{22}
\end{array}\right) .
$$


Converting this in the $\mathrm{K} 1$ formulation, we have the matrix

$$
K_{1}=\left(\begin{array}{cccc}
I_{11}^{r} & -I_{11}^{t} & 0 & 0 \\
I_{11}^{t} & I_{11}^{r} & 0 & 0 \\
I_{21}^{r} & -I_{21}^{i} & I_{22}^{r} & -I_{22}^{t} \\
I_{21}^{t} & I_{21}^{r} & I_{22}^{t} & I_{22}^{r}
\end{array}\right)
$$

The matrix $\mathrm{K}_{1}$ clearly shows that the transformation is no longer a lower triangular matrix. This is the reason why we use the same $L$ and $U$. Similar reactions occur for the K4 formulation.

The second way to implement the preconditioner was to simply generate an individual preconditioner for each of the formulations $\mathrm{K} 1, \mathrm{~K} 4$, and $\mathrm{K} 14$. We also tried diagonal preconditioning because some of the problems we were using for computation were not giving right answers with an ILU preconditioner. For problems like M3D2, M4D2 and vm214img45 (see Table 2), the ILU preconditioner was pivoting and permuting large matrices. Hence for these matrices we used a diagonal preconditioner, a weak preconditioner.

\section{PREVIEW OF COMPUTATIONAL RESULTS}

We have used the $\mathrm{K} 1, \mathrm{~K} 4$, and $\mathrm{K} 14$ formulations to solve several complex liner systems, starting with simple 10-by-10 matrices and on up to larger dimension matrices (Table 2). Each large system comes from a real application, and most of these problems are ill conditioned. Hence, preconditioners like Jacobi or block Jacobi are not sufficient to move eigenvalues to a cloud around point $(1,0)$ in the complex plane in order to get a better solution. We found that ILU preconditioning was very effective in obtaining accurate results. Applying this preconditioner made the problems solvable using fewer iterations. However, in this research we came across one situation where a preconditioner could not be applied: if the diagonal values of a unit lower or upper triangular matrix has any zeros then the non-structure array prevented preconditioning.

Details of these results will be reported in a future communication [Part 2: Results, will be in the March 2003 issueEditor].

\section{REFERENCES}

1. S. Balay, W. Gropp. L. Mclnnes, and B. Smith, PETSC 2.0 Users Manual, Technical Report ANL-95/11 - Revision 2.0.22 (Argonne national Laboratory, Argonne, IL, 1998).

2. Ray S. Tuminaro, Michael A. Heroux, Scott A. Hutchinson, and J.N. Shadid, Official Aztec User's Guide, Version 2.1 (Sandia national laboratories, Albuguerque, NM, 1999).

3. David Day and Michael A. Heroux, "solving Complex-Valued Linear systems via Equivalent Real Formulations" SIAM J. Sci. Comput., Vol. 23, No. 2 (2001), pp. 480-498.

4. Y. Saad, Iterative Methods for Sparse Linear Systems (PWS Publishing Company, Boston, MA, USA, 1996).

5. Youcef Saad and Martin H. Schultz, 'GMRES: A generalized minimal residual algorithm for solving nonsymmetric linear systems," SIAM J. Sci. Statist. Comput., Vol. 7, No. 3 (july 1986), pp. 856-869.

\section{College of Saint Benedict} Saint John's University 


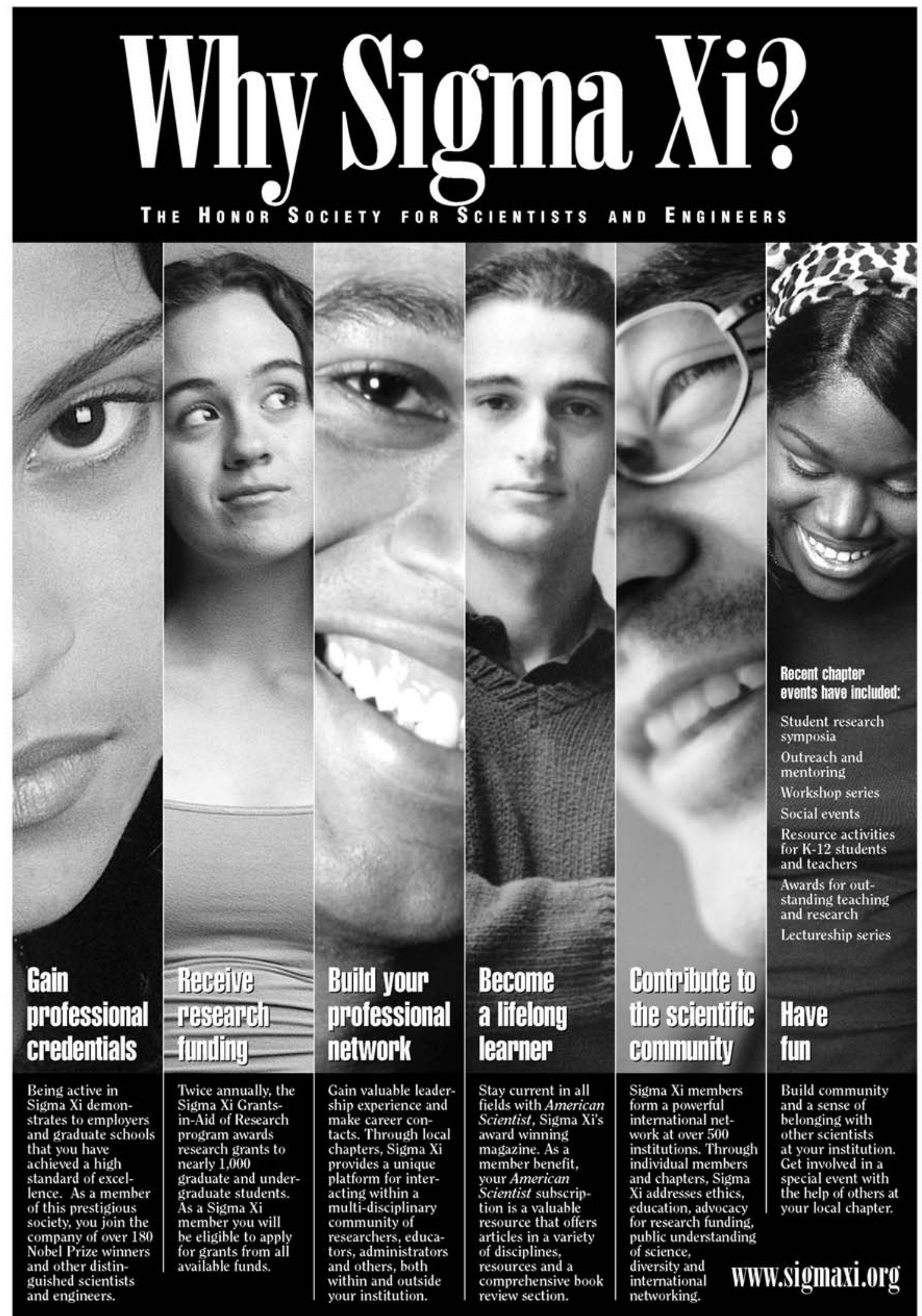

Sigma Xi, The Scientific Research Society • 88 Alexander Drive • P.O. Box 13975 • Research Triangle Park, NC 27708 • 818-549-4691 • 800-243-6534 